\title{
Portal Vein Embolization for Perihilar Cholangiocarcinoma: A Story Worth Repeating
}

\author{
Hop S. Tran Cao, MD, FACS, and Jean-Nicolas Vauthey, MD, FACS \\ Department of Surgical Oncology, The University of Texas MD Anderson Cancer Center, Houston, TX
}

Three decades ago, Makuuchi and colleagues shared with the world their preliminary experience with preoperative portal vein embolization (PVE). ${ }^{1}$ The 14 patients discussed in their report all had perihilar carcinoma (PHC) and would require extended hepatectomy. Although two patients died within 3 months of surgery, the early results were encouraging, demonstrating low rates of postoperative liver failure and bile leak. Olthof et al. ${ }^{2}$ have now retold this compelling story through a retrospective multiinstitutional, multinational cohort study in which 1667 patients with PHC treated at 20 centers were evaluated. Through propensity score matching, the benefits of PVE are clearly demonstrated, with a significant reduction in the rates of postoperative liver failure ( $8 \%$ vs. $36 \%$ ), biliary leakage (10\% vs. $35 \%)$, intra-abdominal abscess (19\% vs. $34 \%$ ), and 90 -day mortality ( $7 \%$ vs. $18 \%$ ). Their rightful conclusion echoed what should by now be a familiar refrain to hepatobiliary surgeons: PVE is an integral component of liver surgery, especially as it pertains to the treatment of PHC. Importantly, the current version of the story also raises some important issues worth addressing.

Due to their anatomic location and propensity for infiltration, PHCs inherently warrant, at the very least, hemihepatectomy, if not extended hepatectomy. While the right liver almost always represents a majority of the total liver volume (TLV), up to $10 \%$ of left livers and $80 \%$ of left lateral sections make up $<20 \%$ of the TLV. ${ }^{3}$ With ample evidence demonstrating high rates of post-hepatectomy liver failure when the future liver remnant (FLR) falls

\footnotetext{
(C) Society of Surgical Oncology 2020
}

First Received: 7 February 2020;

Published Online: 28 February 2020

J.-N. Vauthey, MD, FACS

e-mail: jvauthey@mdanderson.org short of this critical cut-off, it is easy to see why nearly all PVEs in the study were performed for right or extended right hepatectomies. By the same token, it is somewhat disconcerting that nearly a quarter of hepatectomies in the non-PVE cohort were extended right hepatectomies. Certainly, extent of surgery alone does not indicate the need for PVE. Instead, liver volumetry is widely recognized as a critical element in the evaluation of surgical suitability for liver tumors. Our group has previously demonstrated that in patients with PHC, preoperative cholangitis and FLR volume $<30 \%$ (required due to the impaired regenerative potential of the liver in the setting of bile stasis) were major risk factors for postoperative hepatic insufficiency and mortality. ${ }^{4}$ In the current study, a quarter of patients in the non-PVE group had an FLR predicted to measure $<31 \%$ of the TLV. ${ }^{2}$ Subjecting PHC patients to extended right hepatectomies with FLRs in the 20-30\% range, especially in the context of possible cholangitis, is imprudent at best and should be avoided. Instead, efforts at augmenting the FLR must first be undertaken.

In the decades since PVE was first introduced, great strides have been made to potentiate its effectiveness and predict its impact on post-hepatectomy outcomes through measurements of dynamic hypertrophy values. ${ }^{5,6}$ Moreover, PVE has been shown to be extremely safe, with no mortality and low morbidity, ${ }^{7}$ yet its application remains haphazard, even in high-volume centers. The reasons for this are unclear, but, as can be evidenced in the current work, need to be investigated. Among 16 high-volume centers where hepatectomies were performed for PHC in this study, 6 utilized PVE $<10 \%$ of the time, including 4 where the rate was $<5 \%$ (one center did not perform a single PVE). In the face of resounding data showing poor outcomes with small FLRs, one must question whether institutions where PVE is not more routinely available are well-equipped to optimally treat PHCs. While some may 
argue that other methods such as associating liver partition and portal vein ligation for staged hepatectomy (ALPPS) offer viable alternatives to PVE in addressing small FLRs, there is a paucity of data to assess their performance in the treatment of PHC. What little data does exist argues strongly against the use of ALPPS for PHC due to the unacceptably high rate of mortality approaching 50\%, even at high-volume centers. ${ }^{8}$

Beyond the obvious primary benefit of FLR hypertrophy induction, PVE comes with an important hidden benefit that is not well revealed in the current work. Specifically, PVE offers surgeons a chance at better patient and liver selection for major hepatectomy. Indeed, failure to achieve adequate hypertrophy following PVE is a marker of poor hepatic reserve and a predictor of post-hepatectomy liver failure. At the same time, interval disease progression betrays an aggressive tumor biology for which surgical resection would have been a futile endeavor. Perhaps as much as it is a therapeutic tool, PVE serves a diagnostic and prognostic purpose in the management of patients with hepatobiliary malignancies, especially PHC. Thus, it is encouraging to see that the application of PVE for PHC has risen over the study period, and the work shared here by Olthof and colleagues will go a long way in further buttressing this trend. ${ }^{2}$

FUNDING This research was supported in part by the National Institutes of Health through MD Anderson Cancer Center Support Grant CA016672.

DISCLOSURES Hop S. Tran Cao and Jean-Nicolas Vauthey have no disclosures to declare.

\section{REFERENCES}

1. Makuuchi M, Thai BL, Takayasu K, et al. Preoperative portal embolization to increase safety of major hepatectomy for hilar bile duct carcinoma: a preliminary report. Surgery. 1990;107(5):521-7.

2. Olthof PB, Aldrighetti L, Alikhanov R, et al. Portal vein embolization is associated with reduced liver failure and mortality in high-risk resections for perihilar cholangiocarcinoma. Ann Surg Oncol. (In press).

3. Abdalla EK, Denys A, Chevalier P, Nemr RA, Vauthey JN. Total and segmental liver volume variations: implications for liver surgery. Surgery. 2004;135(4):404-10.

4. Ribero D, Zimmitti G, Aloia TA, et al. Preoperative cholangitis and future liver remnant volume determine the risk of liver failure in patients undergoing resection for hilar cholangiocarcinoma. $J$ Am Coll Surg. 2016;223(1):87-97.

5. Kishi Y, Madoff DC, Abdalla EK, et al. Is embolization of segment 4 portal veins before extended right hepatectomy justified? Surgery. 2008;144(5):744-51.

6. Shindoh J, Truty MJ, Aloia TA, et al. Kinetic growth rate after portal vein embolization predicts posthepatectomy outcomes: toward zero liver-related mortality in patients with colorectal liver metastases and small future liver remnant. J Am Coll Surg. 2013;216(2):201-9.

7. Abulkhir A, Limongelli P, Healey AJ, et al. Preoperative portal vein embolization for major liver resection: a meta-analysis. Ann Surg. 2008;247:49-57.

8. Olthof PB, Coelen RJS, Wiggers JK, et al. High mortality after ALPPS for perihilar cholangiocarcinoma: case-control analysis including the first series from the international ALPPS registry. HPB (Oxford). 2017;19(5):381-7.

Publisher's Note Springer Nature remains neutral with regard to jurisdictional claims in published maps and institutional affiliations. 